PROCEEDINGS OF THE

AMERICAN MATHEMATICAL SOCIETY

Volume 129, Number 9, Pages 2715-2726

S 0002-9939(01)05833-6

Article electronically published on February 15, 2001

\title{
THE DISCRETE PRÜFER TRANSFORMATION
}

\author{
MARTIN BOHNER AND ONDŘEJ DOŠLÝ \\ (Communicated by Carmen C. Chicone)
}

\begin{abstract}
The classical Prüfer transformation has proved to be a useful tool in the study of Sturm-Liouville theory. In this paper we introduce the Prüfer transformation for self-adjoint difference equations and use it to obtain oscillation criteria and other results. We then offer an extension of this approach to the case of general symplectic systems on time scales. Time scales have been introduced in order to unify discrete and continuous analysis, and indeed our results cover as special cases both the Prüfer transformation for differential and for difference equations.
\end{abstract}

\section{INTRODUCTION}

The Prüfer transformation (established by H. Prüfer in [19]) is a useful tool in the qualitative theory of second order Sturm-Liouville differential equations

$$
\left(r(t) x^{\prime}\right)^{\prime}+p(t) x=0
$$

where $r$ and $p$ are continuous with $r(t)>0$. By this transformation, any nontrivial solution $x$ and its quasiderivative $r x^{\prime}$ can be expressed in the form

$$
x(t)=\varrho(t) \sin \varphi(t), \quad r(t) x^{\prime}(t)=\varrho(t) \cos \varphi(t),
$$

where $\varrho$ and $\varphi$ satisfy the first order system

$$
\varrho^{\prime}=\cos \varphi(t) \sin \varphi(t)\left(\frac{1}{r(t)}-p(t)\right) \varrho, \quad \varphi^{\prime}=p(t) \sin ^{2} \varphi+\frac{1}{r(t)} \cos ^{2} \varphi .
$$

Since 1926, when the original paper of Prüfer appeared, the Prüfer transformation was extended in various directions. In 1979, Elbert 11] showed that any nontrivial solution $x$ of the so-called half-linear second order differential equation

$$
\left(r(t)\left|x^{\prime}\right|^{\alpha-2} x^{\prime}\right)^{\prime}+p(t)|x|^{\alpha-2} x=0, \quad \alpha>1
$$

(observe that (1.1) is the special case $\alpha=2$ in (1.3)), can be expressed in the form

$$
x(t)=\varrho(t) S(\varphi(t)), \quad r^{1-\beta}(t) x^{\prime}(t)=\varrho(t) S^{\prime}(\varphi(t)),
$$

Received by the editors September 2, 1999 and, in revised form, January 24, 2000.

2000 Mathematics Subject Classification. Primary 39A12; Secondary 39A11, 34K11.

Key words and phrases. Prüfer transformation, Sturm-Liouville difference equations, linear Hamiltonian difference systems, time scales, symplectic systems.

The research of the first author was supported by the University of Missouri Research Board. The research of the second author was supported by the Grant G201/98/0677 of the Czech Grant Agency (Prague). 
where $\beta$ is the conjugate number of $\alpha$, i.e., $\frac{1}{\alpha}+\frac{1}{\beta}=1, S=S(t)$ is the solution of the special half-linear equation

$$
\left(\left|S^{\prime}\right|^{\alpha-2} S^{\prime}\right)^{\prime}+(\alpha-1)|S|^{\alpha-2} S=0, \quad S(0)=0, S^{\prime}(0)=1,
$$

and $\varrho, \varphi$ solve a certain first order differential system similar to (1.2).

Another extension of the Prüfer transformation is given in the papers of Barrett [6] and Reid 20, 21. Here the Prüfer transformation is established for second order self-adjoint matrix differential systems and for linear Hamiltonian systems. The key role in this extension is played by a so-called trigonometric system. A trigonometric system is the first order system

$$
S^{\prime}=\mathcal{Q}(t) C, \quad C^{\prime}=-\mathcal{Q}(t) S,
$$

where $S, C, Q$ are $n \times n$ matrices and $\mathcal{Q}$ is symmetric. The terminology trigonometric system is justified by the fact that in the scalar case $n=1, S(t)=\sin \int^{t} \mathcal{Q}(s) d s$, $C(t)=\cos \int^{t} \mathcal{Q}(s) d s$. In the matrix case (1.4) cannot in general be solved explicitly, but its solutions have many of the properties of classical sine and cosine functions (see [9, 13, 14]). Consider now the linear Hamiltonian system

$$
X^{\prime}=\mathcal{A}(t) X+\mathcal{B}(t) U, \quad U^{\prime}=\mathcal{C}(t) X-\mathcal{A}^{T}(t) U,
$$

where $\mathcal{A}, \mathcal{B}, \mathcal{C}, X, U$ are $n \times n$ matrices and $\mathcal{B}, \mathcal{C}$ are symmetric. Reid [20] showed that there exist a nonsingular differentiable matrix $H$ and a symmetric matrix $\mathcal{Q}$ such that any conjoined basis of (1.5) (i.e., a solution satisfying $X^{T} U-U^{T} X \equiv 0$ and $\left.\operatorname{rank}\left(\begin{array}{l}X \\ U\end{array}\right) \equiv n\right)$ can be expressed in the form

$$
X(t)=S^{T}(t) H(t), \quad U(t)=C^{T}(t) H(t),
$$

where $(S, C)$ is a solution of (1.4) satisfying

$$
S^{T}(t) S(t)+C^{T}(t) C(t)=I, \quad S^{T}(t) C(t)-C^{T}(t) S(t)=0 .
$$

The setup of this paper is as follows. In the next section we introduce the Prüfer transformation for self-adjoint difference equations of second order. We derive discrete analogues for the equations (1.2) and use them to prove the socalled reciprocity principle for Sturm-Liouville difference equations of second order. As another application of our discrete Prüfer transformation we offer an alternative proof of the Leighton-Wintner criterion for oscillation of Sturm-Liouville difference equations, and the last statement of Section 2 is a new oscillation criterion for these equations. The final section features a short introduction into the theory of time scales which has been created by Hilger [16] in order to unify discrete and continuous analysis. Next, we use concepts from the theory of time scales to introduce a Prüfer transformation for such so-called dynamic equations on time scales. A time scale is a closed subset of the reals, and if the time scale is equal to all real numbers, then our Prüfer transformation is the usual Prüfer transformation introduced in [19]. If the time scale is equal to all integers, then our Prüfer transformation is the discrete Prüfer transformation introduced in Section 2 of this paper. At the same time this last section offers extensions of the theory to the symplectic systems case that contains as special cases linear Hamiltonian systems, self-adjoint vector equations, and Sturm-Liouville equations of higher order. 


\section{THE DISCRETE SCALAR CASE AND APPLICATIONS}

In this section we consider a Sturm-Liouville difference equation of second order

$$
\Delta\left(r_{k} \Delta x_{k}\right)+p_{k} x_{k+1}=0,
$$

where $r_{k}$ and $p_{k}$ are real numbers with $r_{k} \neq 0$ for all integers $k$, and where $\Delta$ is the usual forward difference operator defined by $\Delta x_{k}=x_{k+1}-x_{k}$.

Let $x$ be a nontrivial solution of (2.1). Then $x_{k}^{2}+\left(r_{k} \Delta x_{k}\right)^{2} \neq 0$ for all integers $k$ (note that otherwise $x$ would be identically zero) and we can find real numbers $\varrho_{k}>0$ and $\varphi_{k}$ with $0 \leq \Delta \varphi_{k}<2 \pi$ such that the equations

$$
\begin{gathered}
x_{k}=\varrho_{k} \sin \varphi_{k}, \\
r_{k} \Delta x_{k}=\varrho_{k} \cos \varphi_{k}
\end{gathered}
$$

are satisfied for all integers $k$. We call (2.2) and (2.3) the discrete Prüfer transformation. Our first result is a discrete analogue of the equations (1.2), and it reads as follows.

Theorem 2.1. If $x$ is a nontrivial solution of (2.1) and if $\varrho$ and $\varphi$ are defined by (2.2) and (2.3), then the equations

$$
\begin{gathered}
\Delta \varrho_{k}=\varrho_{k}\left[\frac{1}{r_{k}} \cos \varphi_{k} \sin \varphi_{k+1}-p_{k} \sin \varphi_{k} \cos \varphi_{k+1}-\frac{p_{k}}{r_{k}} \cos \varphi_{k} \cos \varphi_{k+1}\right. \\
\left.-\frac{\left(\Delta \sin \varphi_{k}\right)^{2}+\left(\Delta \cos \varphi_{k}\right)^{2}}{2}\right] \\
\sin \Delta \varphi_{k}=\frac{1}{r_{k}} \cos \varphi_{k} \cos \varphi_{k+1}+p_{k} \sin \varphi_{k} \sin \varphi_{k+1}+\frac{p_{k}}{r_{k}} \cos \varphi_{k} \sin \varphi_{k+1}
\end{gathered}
$$

hold true.

Proof. Use of the discrete product rule for (2.2) yields

$$
\begin{aligned}
\sin \varphi_{k+1} \Delta \varrho_{k}+\varrho_{k} \Delta \sin \varphi_{k} & =\Delta\left[\varrho_{k} \sin \varphi_{k}\right]=\Delta x_{k} \\
& =\frac{1}{r_{k}}\left[r_{k} \Delta x_{k}\right]=\frac{1}{r_{k}} \varrho_{k} \cos \varphi_{k},
\end{aligned}
$$

while doing the same for (2.3) implies

$$
\begin{aligned}
\cos \varphi_{k+1} \Delta \varrho_{k}+\varrho_{k} \Delta \cos \varphi_{k} & =\Delta\left[\varrho_{k} \cos \varphi_{k}\right]=\Delta\left[r_{k} \Delta x_{k}\right] \\
& =-p_{k} x_{k+1}=-\frac{p_{k}}{r_{k}}\left[r_{k} \Delta x_{k}\right]-p_{k} x_{k} \\
& =-\frac{p_{k}}{r_{k}} \varrho_{k} \cos \varphi_{k}-p_{k} \varrho_{k} \sin \varphi_{k},
\end{aligned}
$$

where we have also used that $x$ is a solution of (2.1). Hence we obtain the two equations

$$
\begin{gathered}
\sin \varphi_{k+1} \Delta \varrho_{k}+\varrho_{k} \Delta \sin \varphi_{k}=\frac{\varrho_{k}}{r_{k}} \cos \varphi_{k} \\
\cos \varphi_{k+1} \Delta \varrho_{k}+\varrho_{k} \Delta \cos \varphi_{k}=-\frac{p_{k}}{r_{k}} \varrho_{k} \cos \varphi_{k}-p_{k} \varrho_{k} \sin \varphi_{k}
\end{gathered}
$$

We now multiply (2.6) by $\sin \varphi_{k+1}$ and (2.7) by $\cos \varphi_{k+1}$ and add the resulting equations to obtain (2.4). To verify (2.5), we multiply (2.6) by $\cos \varphi_{k+1}$ and (2.7) by $-\sin \varphi_{k+1}$ and add the resulting equations. Dividing the obtained equation by $\varrho_{k}>0$ directly yields (2.5). 
Remark 2.2. Theorem 2.1 suggests a method to construct solutions of the selfadjoint difference equation (2.1): Observe that the difference equation (2.5) for $\varphi$ is independent of $\varrho$. Of course, it might be difficult to solve this equation, but once a solution $\tilde{\varphi}$ of (2.5) is obtained, the linear difference equation (2.4) for $\varrho$ is readily solved.

We proceed to give three applications of the discrete Prüfer transformation introduced in (2.2) and (2.3). The first one is the so-called reciprocity principle that says that a solution $x$ of (2.1) is eventually of one sign (i.e., there exists an integer $N$ such that $x_{k} x_{k+1}>0$ for all $k \geq N$ ) iff its quasiderivative $r \Delta x$ is eventually of one sign. The second application is a discrete version of the Leighton-Wintner criterion (see e.g., [15, pp. 333-335]) that gives a sufficient condition for oscillatory behavior of (2.1) and the third one is a new oscillation/nonoscillation criterion for (2.1). Recall that equation (2.1) is called nonoscillatory if all of its solutions have only finitely many generalized zeros. In the case when $r_{k}>0$ (which we consider here), a solution $x$ of (2.1) is said to have a generalized zero at $m$ provided $x_{m} x_{m+1} \leq 0$. For both applications we assume

$$
p_{k} \quad \text { and } \quad r_{k} \text { are positive for all integers } k \text {. }
$$

We need an auxiliary result which we will prove first.

Lemma 2.3. Assume (2.8), let $x$ be a solution of (2.1), and define $\varphi$ by the Prüfer transformation as before. If $x_{k} x_{k+1}>0$ for some integer $k$, then $0<\Delta \varphi_{k}<\pi$.

Proof. First of all use (2.1), (2.2), and (2.3) to obtain

$$
\cos \varphi_{k+1}+p_{k} \sin \varphi_{k+1}=\frac{r_{k+1} \Delta x_{k+1}+p_{k} x_{k+1}}{\varrho_{k+1}}=\frac{r_{k} \Delta x_{k}}{\varrho_{k+1}}=\frac{\varrho_{k} \cos \varphi_{k}}{\varrho_{k+1}} .
$$

This implies by (2.2) and (2.5)

$$
\begin{aligned}
\sin \Delta \varphi_{k} & =p_{k} \sin \varphi_{k} \sin \varphi_{k+1}+\frac{\cos \varphi_{k}}{r_{k}}\left[\cos \varphi_{k+1}+p_{k} \sin \varphi_{k+1}\right] \\
& =\frac{p_{k} x_{k} x_{k+1}}{\varrho_{k} \varrho_{k+1}}+\frac{\varrho_{k} \cos ^{2} \varphi_{k}}{r_{k} \varrho_{k+1}}>0
\end{aligned}
$$

and hence (observe that by definition $0 \leq \Delta \varphi_{k}<2 \pi$ ) we have $0<\Delta \varphi_{k}<\pi$, which is our desired conclusion.

Theorem 2.4 (Reciprocity Principle). Assume (2.8) and let $x$ be a solution of (2.1). If $x$ is eventually of one sign, then so is $r \Delta x$.

Proof. Let $x$ be a solution of (2.1) and define $\varphi$ by the Prüfer transformation as before. Let $N$ be an integer such that $x_{k} x_{k+1}>0$ for all $k \geq N$. Then the points $\left(r_{k} \Delta x_{k}, x_{k}\right)$ are either in the upper half plane for all $k \geq N$ or in the lower half plane for all $k \geq N$. This and Lemma 2.3 imply

$$
\varphi_{k} \leq \varphi_{N}+\pi \text { for all } k \geq N .
$$

By definition $\varphi_{k} \leq \varphi_{k+1}$ for all $k \geq N$ so that $\lim _{k \rightarrow \infty} \varphi_{k}$ must exist. But then also $\lim _{k \rightarrow \infty} \cos \varphi_{k}$ exists and hence (observe also that $\cos \varphi_{k} \geq \cos \varphi_{k+1}$ for all $k \geq N$ or $\cos \varphi_{k} \leq \cos \varphi_{k+1}$ for all $\left.k \geq N\right)$ there exists an integer $M$ such that $\cos \varphi_{k} \cos \varphi_{k+1}>0$ for all $k \geq M$. This together with (2.3) proves our claim. 
Remark 2.5. The converse implication of Theorem [2.4 is true as well, and this follows from the fact that $\tilde{x}=r \Delta x$ satisfies the so-called reciprocal equation

$$
\Delta\left(\frac{1}{p_{k}} \Delta \tilde{x}_{k}\right)+\frac{1}{r_{k+1}} \tilde{x}_{k+1}=0
$$

and one can use the same argumentation as in the proof of Theorem 2.4

Theorem 2.6 (Leighton-Wintner Criterion). Assume (2.8) and suppose

$$
\sum_{k=0}^{\infty} \frac{1}{r_{k}}=\sum_{k=0}^{\infty} p_{k}=\infty
$$

Then the equation (2.1) is oscillatory.

Proof. Let (2.10) be satisfied. For the sake of contradiction we assume that (2.1) is not oscillatory. Then there exists a solution $x$ of (2.1) that is eventually either of positive or of negative sign. We define now $\varrho$ and $\varphi$ via the Prüfer transformation from (2.2) and (2.3). By Lemma 2.3 we have $0<\Delta \varphi_{k}<\pi$. Hence $\varphi$ must be bounded above (otherwise $x$ would change its sign infinitely many times). Therefore $\lim _{k \rightarrow \infty} \varphi_{k}=: \varphi_{\infty}$ exists and hence both

$$
\lim _{k \rightarrow \infty} \sin \varphi_{k}=: \alpha \text { and } \lim _{k \rightarrow \infty} \cos \varphi_{k}=: \beta
$$

exist as well. Furthermore $\alpha^{2}+\beta^{2}=1$ so that at least one of the numbers $\alpha$ and $\beta$ is not zero. First we assume $\alpha \neq 0$. Then there exists an integer $M \geq N$ such that $\sin \varphi_{k} \neq 0$ for all $k \geq M$. Let $k \geq M$. Then we have by (2.5)

$$
\begin{aligned}
-\Delta \cot \varphi_{k} & =\frac{\sin \Delta \varphi_{k}}{\sin \varphi_{k} \sin \varphi_{k+1}}=p_{k}+\frac{\cos \varphi_{k}\left[\cos \varphi_{k+1}+p_{k} \sin \varphi_{k+1}\right]}{r_{k} \sin \varphi_{k} \sin \varphi_{k+1}} \\
& =p_{k}+\frac{\varrho_{k}^{2} \cos ^{2} \varphi_{k}}{r_{k} x_{k} x_{k+1}} \geq p_{k}
\end{aligned}
$$

(apply also (2.9) ) and hence

$$
\cot \varphi_{k+1}-\cot \varphi_{M} \leq-\sum_{i=M}^{k} p_{i}
$$

But letting $k \rightarrow \infty$ we see that the left hand side of the above inequality converges to $\cot \varphi_{\infty}-\cot \varphi_{M} \in \mathbb{R}$ while by (2.10) the right hand side tends to $-\infty$, a contradiction. Similarly, when $\beta \neq 0$, there exists an integer $M>N$ such that $\cos \varphi_{k} \neq 0$ for all $k \geq M$. Let $k \geq M$. Then we have by (2.5)

$$
\begin{aligned}
\Delta \tan \varphi_{k} & =\frac{\sin \Delta \varphi_{k}}{\cos \varphi_{k} \cos \varphi_{k+1}}=\frac{1}{r_{k}}+\frac{p_{k} \sin \varphi_{k+1}\left[\sin \varphi_{k}+\frac{1}{r_{k}} \cos \varphi_{k}\right]}{\cos \varphi_{k} \cos \varphi_{k+1}} \\
& =\frac{1}{r_{k}}+\frac{\varrho_{k}^{2} \varrho_{k+1}^{2} p_{k} \sin ^{2} \varphi_{k+1}}{\left(r_{k} \Delta x_{k}\right)\left(r_{k+1} \Delta x_{k+1}\right)} \geq \frac{1}{r_{k}}
\end{aligned}
$$

by the reciprocity principle from Theorem 2.4. Again, as before, this contradicts (2.10). The proof of the statement is complete.

At the end of this section we present a new oscillation and nonoscillation criterion for (2.1). This criterion could be proved using the material of this section, however an easier proof can be given based on the considerations of the next section, so we postpone the proof of the next statement to the end of the paper. 
Theorem 2.7. Suppose that

$$
0<p_{k}<4 r_{k} \quad \text { for large } k .
$$

Equation (2.1) is nonoscillatory provided

$$
\sum^{\infty} \operatorname{arccot}\left\{\frac{1}{p_{k}}-\frac{r_{k} p_{k}}{\sqrt{p_{k}^{2}+\left(1-p_{k} r_{k}\right)^{2}}-1+r_{k} p_{k}-\frac{p_{k}^{2}}{2}}\right\}<\infty,
$$

and it is oscillatory if

$$
\sum^{\infty} \operatorname{arccot}\left\{\frac{1}{p_{k}}+\frac{r_{k} p_{k}}{\sqrt{p_{k}^{2}+\left(1-p_{k} r_{k}\right)^{2}}+1-r_{k} p_{k}+\frac{p_{k}^{2}}{2}}\right\}=\infty .
$$

\section{Time scales And the systems CASE}

In this section we present a further extension of the Prüfer transformation to so-called symplectic dynamic systems on time scales. This extension incorporates both the continuous and discrete Prüfer transformation.

A time scale $\mathbb{T}$ is any closed subset of the reals $\mathbb{R}$. This concept was introduced by Hilger 16 (an alternative terminology for time scale is measure chain). There and in subsequent papers [2, 5, 12, 17], the calculus on time scales has been developed, which in the case $\mathbb{T}=\mathbb{R}$ reduces to the usual differential and integral calculus and in the case $\mathbb{T}=\mathbb{Z}$ to the calculus of differences and sums.

Recall first some basic concepts of the time scale calculus. We define the jump operators and graininess by

$$
\sigma(t)=\inf \{s>t, s \in \mathbb{T}\}, \quad \rho(t)=\sup \{s<t, s \in \mathbb{T}\}, \quad \mu(t)=\sigma(t)-t
$$

(supplemented by $\inf \emptyset=\sup \mathbb{T}$ and $\sup \emptyset=\inf \mathbb{T}$ ). Further, for a function $f: \mathbb{T} \rightarrow$ $\mathbb{R}$ (or generally with values in any Banach space) the generalized derivative $f^{\Delta}(t)$ at a point $t \in \mathbb{T}$ is defined provided that for all $\varepsilon>0$ there exists a neighborhood $\mathcal{U}$ of $t$ in $\mathbb{T}$ such that

$$
\left|f(\sigma(t))-f(s)-f^{\Delta}(t)(\sigma(t)-s)\right| \leq \varepsilon|\sigma(t)-s| \text { holds for all } s \in \mathcal{U},
$$

and then the formula $f(\sigma(t))=f(t)+\mu(t) f^{\Delta}(t)$ holds for all $t \in \mathbb{T}$. If $\mathbb{T}=\mathbb{R}$, then $f^{\Delta}=f^{\prime}$ is the usual derivative and if $\mathbb{T}=\mathbb{Z}$, then $f^{\Delta}=\Delta f$ is the usual forward difference. A function $f$ is said to be rd-continuous if $f$ is continuous in all points $t$ with $\sigma(t)=t$ and if $\lim _{s \rightarrow t-} f(x)$ exists in all points $t$ with $\rho(t)=t$. Similarly, $f$ is called rd-continuously differentiable if $f^{\Delta}$ exists and is rd-continuous. For $f \circ \sigma$ we write $f^{\sigma}$ and we will omit the argument $t$ of time scale functions when no ambiguity may arise. If $A$ and $B$ are matrix-valued differentiable functions on $\mathbb{T}$, then $(A+B)^{\Delta}=A^{\Delta}+B^{\Delta},(A B)^{\Delta}=A^{\Delta} B^{\sigma}+A B^{\Delta}$, and (if $A A^{\sigma}$ is invertible) $\left(A^{-1}\right)^{\Delta}=-\left(A^{-1}\right)^{\sigma} A^{\Delta} A^{-1}$.

A symplectic dynamic system on a time scale $\mathbb{T}$ is a first order linear system

$$
z^{\Delta}=\mathcal{S}(t) z
$$

where $z=\left(\begin{array}{l}x \\ u\end{array}\right): \mathbb{T} \rightarrow \mathbb{R}^{n}$, and where $\mathcal{S}$ is a $2 n \times 2 n$ matrix satisfying (see [10])

$$
\mathcal{S}^{T}(t) \mathcal{J}+\mathcal{J S}(t)+\mu(t) \mathcal{S}^{T}(t) \mathcal{J} \mathcal{S}(t)=0
$$

with $\mathcal{J}=\left(\begin{array}{cc}0 & I \\ -I & 0\end{array}\right)$. This system incorporates the linear Hamiltonian differential system (1.5) if $\mathbb{T}=\mathbb{R}$ since then $\mu(t)=0$, i.e., (3.2) reduces to $\mathcal{S}^{T} \mathcal{J}+\mathcal{J} \mathcal{S}=0$, and this immediately implies that $\mathcal{S}$ is of the form $\mathcal{S}=\left(\begin{array}{cc}\mathcal{A} & \mathcal{B} \\ \mathcal{C} & -\mathcal{A}^{T}\end{array}\right)$ with symmetric 
$\mathcal{B}, \mathcal{C}$. On the other hand, if $\mathbb{T}=\mathbb{Z}$, then (3.1) is the so-called symplectic difference system whose basic oscillatory properties were established in the recent paper [7]. A symplectic difference system is therefore a first order system of the form

$$
z_{k+1}=\overline{\mathcal{S}}_{k} z_{k}, \quad \overline{\mathcal{S}}_{k}=I+\mathcal{S}_{k},
$$

where the matrix $\overline{\mathcal{S}}$ is symplectic, i.e., $\overline{\mathcal{S}}^{T} \mathcal{J} \overline{\mathcal{S}}=\mathcal{J}$. Symplectic difference systems cover a large variety of difference equations and systems, among them also the Sturm-Liouville difference equation (2.1) since substituting $u=r \Delta x$ and expanding the forward differences, this equation can be written in the form

$$
\left(\begin{array}{l}
x_{k+1} \\
u_{k+1}
\end{array}\right)=\left(\begin{array}{cc}
1 & \frac{1}{r_{k}} \\
-p_{k} & 1-\frac{p_{k}}{r_{k}}
\end{array}\right)\left(\begin{array}{l}
x_{k} \\
u_{k}
\end{array}\right)
$$

and directly one can verify that the matrix in this system is really symplectic.

Finally recall the concept of a trigonometric dynamic system. This is a symplectic dynamic system of the form (3.1), whose matrix $\mathcal{S}$, in addition to (3.2), satisfies the condition $\mathcal{J}^{T} \mathcal{S} \mathcal{J}=\mathcal{S}$ which means that the transformation $z=\mathcal{J} \bar{z}$ transforms (3.1) into itself.

Remark 3.1. If the matrix $\mathcal{S}$ from system (3.1) satisfies $\mathcal{J}^{T} \mathcal{S} \mathcal{J}=\mathcal{S}$, then (3.1) is a symplectic system if and only if the condition

$$
\mathcal{S}+\mathcal{S}^{T}+\mu \mathcal{S}^{T} \mathcal{S}=0 .
$$

Now we can formulate the main result of our paper, the Prüfer type transformation for symplectic dynamic systems.

Theorem 3.2. Let $Z=\left(\begin{array}{l}X \\ U\end{array}\right)$ be a $2 n \times n$ matrix conjoined basis of (3.1) (i.e., $X^{T} U=U^{T} X$ and $\left.\operatorname{rank}\left(\begin{array}{l}X \\ U\end{array}\right)=n\right)$. Then there exist an $r d$-continuously differentiable nonsingular $n \times n$ matrix $H$ and $n \times n$ matrices $S, C$ such that $\left(\begin{array}{l}X \\ U\end{array}\right)$ can be expressed in the form

$$
X(t)=S^{T}(t) H(t), \quad U(t)=C^{T}(t) H(t),
$$

where $\left(\begin{array}{l}S \\ C\end{array}\right)$ is a solution of the trigonometric dynamic system

$$
\left(\begin{array}{l}
S \\
C
\end{array}\right)^{\Delta}=\left(\begin{array}{cc}
\mathcal{P} & \mathcal{Q} \\
-\mathcal{Q} & \mathcal{P}
\end{array}\right)\left(\begin{array}{l}
S \\
C
\end{array}\right)
$$

satisfying $S^{T} S+C^{T} C=I, S^{T} C-C^{T} S=0$, with

$$
\left\{\begin{array}{l}
\mathcal{P}=\left(H^{\sigma}\right)^{T-1}\left(\begin{array}{l}
X \\
U
\end{array}\right)^{T} \mathcal{S}^{T}\left(\begin{array}{l}
X \\
U
\end{array}\right) H^{-1}-H^{\Delta}, \\
\mathcal{Q}=\left(H^{\sigma}\right)^{T-1}\left(\begin{array}{l}
X \\
U
\end{array}\right)^{T} \mathcal{S}^{T} \mathcal{J}\left(\begin{array}{l}
X \\
U
\end{array}\right) H^{-1},
\end{array}\right.
$$

and $H$ solves the first order system

$$
H^{\Delta}=\left(\tilde{Z}^{\sigma}\right)^{T}\left(\mathcal{S} \tilde{Z}-\tilde{Z}^{\Delta}\right) H,
$$

where $\tilde{Z}=\left(\begin{array}{l}S^{T} \\ C^{T}\end{array}\right)$. Moreover, we have

$$
\left(\tilde{Z}^{\sigma}\right)^{T} \mathcal{J}\left(\mathcal{S} \tilde{Z}-\tilde{Z}^{\Delta}\right)=0 .
$$

Proof. Let $Z=\left(\begin{array}{l}X \\ U\end{array}\right)$ be a conjoined basis of (3.1) and let $H$ be any rd-continuously differentiable matrix satisfying $H^{T} H=X^{T} X+U^{T} U=(X+\mathrm{i} U)^{*}(X+\mathrm{i} U)$ (here * stands for the conjugate transpose of the matrix indicated). The existence of such 
a matrix can be proved in the same way as in the continuous case $\mathbb{T}=\mathbb{R}$ (see $[6$ ] Lemma 3.1]). Denote $\mathcal{Z}=(X+\mathrm{i} U) H^{-1}$. Then

$$
\begin{aligned}
\mathcal{Z}^{\Delta} & =\left(X^{\Delta}+\mathrm{i} U^{\Delta}\right)\left(H^{\sigma}\right)^{-1}-(X+\mathrm{i} U) H^{-1} H^{\Delta}\left(H^{\sigma}\right)^{-1} \\
& =\mathcal{Z}\left[\mathcal{Z}^{-1}(X+\mathrm{i} U)^{*-1}(X+\mathrm{i} U)^{*}\left(X^{\Delta}+\mathrm{i} U^{\Delta}\right)-H^{\Delta}\right]\left(H^{\sigma}\right)^{-1} \\
& =\mathcal{Z}\left[H\left(H^{T} H\right)^{-1}\left(X^{T}-\mathrm{i} U^{T}\right)\left(X^{\Delta}+\mathrm{i} U^{\Delta}\right)-H^{\Delta}\right]\left(H^{\sigma}\right)^{-1} \\
& =\mathcal{Z} H^{T-1}\left[X^{T} X^{\Delta}+U^{T} U^{\Delta}-H^{T} H^{\Delta}+\mathrm{i}\left(X^{T} U^{\Delta}-U^{T} X^{\Delta}\right)\right]\left(H^{\sigma}\right)^{-1} \\
& =\mathcal{Z} H^{T-1}\left[\left(\begin{array}{l}
X \\
U
\end{array}\right)^{T} \mathcal{S}\left(\begin{array}{l}
X \\
U
\end{array}\right)-H^{T} H^{\Delta}+\mathrm{i}\left(\begin{array}{l}
X \\
U
\end{array}\right)^{T} \mathcal{J} \mathcal{S}\left(\begin{array}{l}
X \\
U
\end{array}\right)\right]\left(H^{\sigma}\right)^{-1} \\
& =\mathcal{Z}\left(\mathcal{P}^{T}-\mathrm{i} \mathcal{Q}^{T}\right),
\end{aligned}
$$

i.e., $\left(\mathcal{Z}^{T}\right)^{\Delta}=(\mathcal{P}-\mathrm{i} \mathcal{Q}) \mathcal{Z}^{T}$, where $\mathcal{P}, \mathcal{Q}$ are given by (3.8) (observe that $\mathcal{J}^{T}=-\mathcal{J}$ ). Now, if we denote $S^{T}=X H^{-1}, C^{T}=U H^{-1}$, then the last system can be written in the form

$$
\left(\begin{array}{l}
S \\
C
\end{array}\right)^{\Delta}=\tilde{\mathcal{S}}\left(\begin{array}{l}
S \\
C
\end{array}\right), \quad \tilde{\mathcal{S}}=\left(\begin{array}{cc}
\mathcal{P} & \mathcal{Q} \\
-\mathcal{Q} & \mathcal{P}
\end{array}\right)
$$

and this immediately implies that $\mathcal{J}^{T} \tilde{\mathcal{S}} \mathcal{J}=\tilde{\mathcal{S}}$. Further, the fact that $\mathcal{Z}$ is unitary gives

$$
\begin{aligned}
0 & =\left(\mathcal{Z} \mathcal{Z}^{*}\right)^{\Delta}=\mathcal{Z}^{\Delta}\left(\mathcal{Z}^{*}+\mu \mathcal{Z}^{*^{\Delta}}\right)+\mathcal{Z} \mathcal{Z}^{*^{\Delta}} \\
& =\mathcal{Z}\left(\mathcal{P}^{T}-\mathrm{i} \mathcal{Q}^{T}\right)\left[\mathcal{Z}^{*}+\mu(\mathcal{P}+\mathrm{i} \mathcal{Q}) \mathcal{Z}^{*}\right]+\mathcal{Z}(\mathcal{P}+\mathrm{i} \mathcal{Q}) \mathcal{Z}^{*} \\
& =\mathcal{Z}\left[\mathcal{P}^{T}-\mathrm{i} \mathcal{Q}^{T}+\mu\left(\mathcal{P}^{T}-\mathrm{i} \mathcal{Q}^{T}\right)(\mathcal{P}+\mathrm{i} \mathcal{Q})+\mathcal{P}+\mathrm{i} \mathcal{Q}\right] \mathcal{Z}^{*} \\
& =\mathcal{Z}[\tilde{\mathcal{P}}+\mathrm{i} \tilde{\mathcal{Q}}] \mathcal{Z}^{*}
\end{aligned}
$$

with

$$
\tilde{\mathcal{P}}=\mathcal{P}+\mathcal{P}^{T}+\mu\left(\mathcal{P}^{T} \mathcal{P}+\mathcal{Q}^{T} \mathcal{Q}\right), \quad \tilde{\mathcal{Q}}=\mathcal{Q}-\mathcal{Q}^{T}+\mu\left(\mathcal{P}^{T} \mathcal{Q}-\mathcal{Q}^{T} \mathcal{P}\right)
$$

Hence both $\tilde{\mathcal{P}}$ and $\tilde{\mathcal{Q}}$ vanish and therefore

$$
\mathcal{S}+\mathcal{S}^{T}+\mu \mathcal{S}^{T} \mathcal{S}=\left(\begin{array}{cc}
\tilde{\mathcal{P}} & \tilde{\mathcal{Q}} \\
-\tilde{\mathcal{Q}} & \tilde{\mathcal{P}}
\end{array}\right)=0
$$

i.e., (3.1) is really (see Remark 3.1) a symplectic system. Finally, if we denote $\tilde{Z}=Z H^{-1}$, then

$$
\mathcal{S} \tilde{Z} H=\mathcal{S} Z=Z^{\Delta}=(\tilde{Z} H)^{\Delta}=\tilde{Z}^{\sigma} H^{\Delta}+\tilde{Z}^{\Delta} H .
$$

Multiplying (3.12) from the left by $\left(\tilde{Z}^{\sigma}\right)^{T}$ and using the fact that

$$
\tilde{Z}^{T} \tilde{Z}=C C^{T}+S S^{T}=H^{T-1}\left(U^{T} U+X^{T} X\right) H^{-1}=I
$$

we get (3.9). Using

$$
\tilde{Z}^{T} \mathcal{J} \tilde{Z}=S C^{T}-C S^{T}=H^{T-1}\left(X^{T} U-U^{T} X\right) H^{-1}=0
$$

we obtain (3.10) by multiplying (3.12) from the left by $\left(\tilde{Z}^{\sigma}\right)^{T} \mathcal{J}$. 
Remark 3.3. (i) First we show that the Prüfer transformation given in Theorem 3.2 covers the Prüfer transformation of linear Hamiltonian differential systems mentioned in the first section. By Theorem 3.2, if $\mathbb{T}=\mathbb{R}$, the conjoined basis $\left(\begin{array}{l}X \\ U\end{array}\right)$ can be expressed in the form (3.6), where $\left(\begin{array}{l}S \\ C\end{array}\right)$ solves the system

$$
S^{\prime}=\mathcal{P}(t) S+\mathcal{Q}(t) C, \quad C^{\prime}=-\mathcal{Q}(t) S+\mathcal{P}(t) C
$$

with $\mathcal{P}$ antisymmetric $\left(\mathcal{P}^{T}+\mathcal{P}=0\right)$ and $\mathcal{Q}$ symmetric. The transformation $S=G \tilde{S}$, $C=G^{T-1} \tilde{C}$, where $G^{\prime}=\mathcal{P}(t) G$, transforms (3.13) into the system

$$
\tilde{S}^{\prime}=G^{-1}(t) \mathcal{Q}(t) G^{T-1}(t) \tilde{C}, \quad \tilde{C}^{\prime}=-G^{T}(t) \mathcal{Q}(t) G(t) \tilde{S},
$$

and since $G^{-1}=G^{T}$ if $G$ is orthogonal at an initial value, this is really the same trigonometric system (1.5) as considered by Barrett [6] and Reid 20, 21].

(ii) In the discrete case $\mathbb{T}=\mathbb{Z}$ the trigonometric difference system (i.e., a symplectic system (3.3) whose matrix satisfies $\mathcal{J}^{T} \tilde{\mathcal{S}} \mathcal{J}=\tilde{\mathcal{S}}$ ) can be written in the form

$$
\left(\begin{array}{c}
S_{k+1} \\
C_{k+1}
\end{array}\right)=\left(\begin{array}{cc}
\mathcal{P}_{k} & \mathcal{Q}_{k} \\
-\mathcal{Q}_{k} & \mathcal{P}_{k}
\end{array}\right)\left(\begin{array}{l}
S_{k} \\
C_{k}
\end{array}\right)
$$

with the matrices $\mathcal{P}, \mathcal{Q}$ satisfying

$$
\mathcal{P}^{T} \mathcal{Q}-\mathcal{Q}^{T} \mathcal{P}=0, \quad \mathcal{P}^{T} \mathcal{P}+\mathcal{Q}^{T} \mathcal{Q}=I .
$$

A system of this kind was introduced by Anderson [4] (see also [8]). In particular, if $n=1$, then by (3.15) there exists $\psi_{k}$ such that $\mathcal{P}_{k}=\cos \psi_{k}, \mathcal{Q}_{k}=\sin \psi_{k}$, and the solution of (3.14) is of the form

$$
S_{k}=\sin \left(\sum^{k-1} \psi_{j}\right), \quad C_{k}=\cos \left(\sum^{k-1} \psi_{j}\right) .
$$

Consequently, if $\varphi_{k}=\sum^{k-1} \psi_{j}$ and $\varrho_{k}=H_{k}$, we have just rediscovered the Prüfer transformation established in the previous section. Moreover, using the fact that (2.11) can be written as a symplectic difference system (3.4) and by (3.8), we have for $x \neq 0$ that

$$
\cot \Delta \varphi=\frac{x^{2}+\left(\frac{1}{r}-p\right) x r \Delta x+\left(1-\frac{p}{r}\right)(r \Delta x)^{2}}{x^{2} p+\frac{p}{r} x r \Delta x+\frac{1}{r}(r \Delta x)^{2}} .
$$

(iii) Observe that the differential equation for the angle variable $\varphi$ in the classical Prüfer transformation is closely related to the Riccati equation corresponding to (1.1). Indeed, in all points where the solution $x$ of (1.1) is nonzero, the ratio $w=\frac{r x^{\prime}}{x}$ solves the Riccati equation

$$
w^{\prime}+p(t)+\frac{w^{2}}{r(t)}=0 .
$$

Substituting here for $x$ and $r x^{\prime}$ from the Prüfer transformation, i.e., $w=\cot \varphi$, we get just the second equation in (1.2). A similar situation we have in the discrete case. Substituting into the Riccati equation (see 1, 3, 18, ) corresponding to (2.1) we get the equation

$$
\Delta(\cot \varphi)+p_{k}+\frac{\cot ^{2} \varphi}{r_{k}+\cot \varphi}=0 .
$$

However, since the discrete analogue of the chain rule for differentiation is not available, the last equation is more difficult than the second equation in (1.2) since 
we cannot generally "extract" $\Delta \varphi$ from this equation. A good candidate for a difference equation with extracted $\Delta \varphi$ could be equation (3.16). In particular, if (2.11) holds, then the function $\mathcal{Q}$ in the scalar discrete trigonometric system (3.14) is positive as can be verified by a direct computation; hence $\Delta \varphi=\psi \in(0, \pi)$ and (3.16) takes the form (substituting there from (2.2), (2.3)

$$
\Delta \varphi=\operatorname{arccot} \frac{\sin ^{2} \varphi+\left(\frac{1}{r}-p\right) \cos \varphi \sin \varphi+\left(1-\frac{p}{r}\right) \cos ^{2} \varphi}{p \sin ^{2} \varphi+\frac{p}{r} \cos \varphi \sin \varphi+\frac{1}{r} \cos ^{2} \varphi} .
$$

Using this observation we can now easily prove Theorem 2.7

Proof of Theorem 2.7. The proof of the theorem is based on the inequalities

$$
\begin{aligned}
\frac{1}{p}-\frac{r p}{\sqrt{p^{2}+(1-p r)^{2}}-1+r p-\frac{p^{2}}{2}} & \leq \frac{\mu^{2}+\left(\frac{1}{r}-p\right) \mu \nu+\left(1-\frac{p}{r}\right) \nu^{2}}{p \mu^{2}+\frac{p}{r} \mu \nu+\frac{1}{r} \nu^{2}} \\
& \leq \frac{1}{p}+\frac{r p}{\sqrt{p^{2}+(1-p r)^{2}}+1-r p+\frac{p^{2}}{2}}
\end{aligned}
$$

which under (2.11) hold for any $\mu, \nu$ satisfying $\mu^{2}+\nu^{2}=1$. To show this, consider the rational function

$$
F(t)=\frac{t^{2}+b t+c}{\alpha t^{2}+\gamma}
$$

with $\alpha, \gamma>0$ and $b, c \in \mathbb{R}, b \neq 0$. Then

$$
\max _{t \in \mathbb{R}} F(t)=\frac{1}{\alpha}+\frac{b^{2}}{2(\sqrt{D}+\gamma-\alpha c)}, \quad \min _{t \in \mathbb{R}} F(t)=\frac{1}{\alpha}-\frac{b^{2}}{2(\sqrt{D}-\gamma+\alpha c)}
$$

where $D=(\gamma-\alpha c)^{2}+b^{2} \alpha \gamma$. Indeed, since $\lim _{t \rightarrow \pm \infty} F(t)=\frac{1}{\alpha}, F$ attains its supremum and infimum over $\mathbb{R}$ at its stationary points. By differentiation $F^{\prime}(t)=0$ iff

$$
t=t_{1,2}=\frac{\gamma-\alpha c \pm \sqrt{D}}{b \alpha}
$$

and substituting these values into $F$ we get (3.19).

Now, if $\nu \neq 0$ in (3.18), dividing the numerator and denominator of this fraction by $\nu^{2}$ we get the rational function

$$
\frac{\lambda^{2}+\left(\frac{1}{r}-p\right) \lambda+1-\frac{p}{r}}{p \lambda^{2}+\frac{p}{r} \lambda+\frac{1}{r}}=\frac{t^{2}-p t+1-\frac{p}{2 r}-\frac{1}{4 r^{2}}}{p t^{2}+\frac{1}{r}-\frac{p}{4 r^{2}}},
$$

where $\lambda=\frac{\mu}{\nu}, t=\lambda+\frac{1}{2 r}$, and setting

$$
b=-p, \quad c=1-\frac{p}{2 r}-\frac{1}{4 r^{2}}, \quad \alpha=p, \quad \gamma=\frac{1}{r}-\frac{p}{4 r^{2}},
$$

we see that (3.18) really holds. In case $\nu=0$ these inequalities are trivial since

$$
\frac{r p}{\sqrt{p^{2}+(1-p r)^{2}} \pm\left(1-r p+\frac{p^{2}}{2}\right)}>0 .
$$

In the remaining part of the proof we proceed similarly as in the proof of Theorems 2.4 and 2.6. Since $0<\Delta \varphi_{k}<\pi$, equation (2.1) is nonoscillatory if there exists a finite limit

$$
\varphi_{\infty}:=\lim _{k \rightarrow \infty} \varphi_{k}=\sum_{k=0}^{\infty} \Delta \varphi_{k}-\varphi_{0}
$$


and it is oscillatory if $\varphi_{\infty}=\infty$. Now, according to (3.18) we have

$$
\begin{aligned}
& \sum_{k=0}^{\infty} \operatorname{arccot}\left\{\frac{1}{p_{k}}+\frac{r_{k} p_{k}}{\sqrt{p_{k}^{2}+\left(1-p_{k} r_{k}\right)^{2}}+1-r_{k} p_{k}+\frac{p_{k}^{2}}{2}}\right\} \\
& \quad \leq \sum_{k=0}^{\infty} \operatorname{arccot} \frac{\sin ^{2} \varphi_{k}+\left(\frac{1}{r_{k}}-p_{k}\right) \cos \varphi_{k} \sin \varphi_{k}+\left(1-\frac{p_{k}}{r_{k}}\right) \cos ^{2} \varphi_{k}}{p_{k} \sin ^{2} \varphi_{k}+\frac{p_{k}}{r_{k}} \cos \varphi_{k} \sin \varphi_{k}+\frac{1}{r_{k}} \cos ^{2} \varphi_{k}} \\
& \quad=\sum_{k=0}^{\infty} \operatorname{arccot}\left\{\frac{1}{p_{k}}-\frac{r_{k} p_{k}}{\sqrt{p_{k}^{2}+\left(1-p_{k} r_{k}\right)^{2}}-1+r_{k} p_{k}-\frac{p_{k}^{2}}{2}}\right\}
\end{aligned}
$$

but since by (3.17) the expression in the middle equals $\varphi_{\infty}-\varphi_{0}$, this completes the proof.

\section{REFERENCES}

[1] R. P. Agarwal. Difference Equations and Inequalities. Marcel Dekker, Inc., New York, 1992. MR 92m:39002

[2] R. P. Agarwal and M. Bohner. Basic calculus on time scales and some of its applications. Results Math., 35(1-2):3-22, 1999. MR 99m:41030

[3] C. D. Ahlbrandt and A. C. Peterson. Discrete Hamiltonian Systems: Difference Equations, Continued Fractions, and Riccati Equations. Kluwer Academic Publishers, Boston, 1996.

[4] D. Anderson. Discrete trigonometric matrix functions. Panamer. Math. J., 7(1):39-54, 1997. MR 99e:39004

[5] B. Aulbach and S. Hilger. Linear dynamic processes with inhomogeneous time scale. In Nonlinear Dynamics and Quantum Dynamical Systems. Akademie Verlag, Berlin, 1990. MR 91g:58266

[6] J. H. Barrett. A Prüfer transformation for matrix differential equations. Proc. Amer. Math. Soc., 8:510-518, 1957. MR 19:415f

[7] M. Bohner and O. Došlý. Disconjugacy and transformations for symplectic systems. Rocky Mountain J. Math., 27(3):707-743, 1997. MR 99e:39007

[8] M. Bohner and O. Došlý. Trigonometric transformations of symplectic difference systems. $J$. Differential Equations, 163:113-129, 2000. CMP 2000:11

[9] O. Došlý. On some properties of trigonometric matrices. Čas. Pěst. Mat., 112:188-196, 1987. MR 88g:34041

[10] O. Došlý and R. Hilscher. Disconjugacy, transformations and quadratic functionals for symplectic dynamic systems on time scales, 2000, J. Differ. Equations Appl., to appear.

[11] Á. Elbert. A half-linear second order differential equation. Colloq. Math. Soc. János Bolyai, 30:158-180, 1979. MR 84g:34008

[12] L. Erbe and S. Hilger. Sturmian theory on measure chains. Differential Equations Dynam. Systems, 1(3):223-244, 1993. MR 95b:39002

[13] G. J. Etgen. A note on trigonometric matrices. Proc. Amer. Math. Soc., 17:1226-1232, 1966. MR 35:4504

[14] G. J. Etgen. Oscillation properties of certain nonlinear matrix equations of second order. Trans. Amer. Math. Soc., 122:289-310, 1966.

[15] H. Heuser. Gewöhnliche Differentialgleichungen. B. G. Teubner, Stuttgart, 1989. MR 90f:34001

[16] S. Hilger. Analysis on measure chains - a unified approach to continuous and discrete calculus. Results Math., 18:18-56, 1990. MR 91m:26027

[17] S. Hilger. Special functions, Laplace and Fourier transform on measure chains. Dynam. Systems Appl., 8(3-4):471-488, 1999. Special Issue on "Discrete and Continuous Hamiltonian Systems", edited by R. P. Agarwal and M. Bohner. CMP 2000:04

[18] W. G. Kelley and A. C. Peterson. Difference Equations: An Introduction with Applications. Academic Press, San Diego, 1991. MR 93f:39002

[19] H. Prüfer. Neue Herleitung der Sturm-Liouvilleschen Reihenentwicklung stetiger Funktionen. Math. Ann., 95:499-518, 1926. 
[20] W. T. Reid. A Prüfer transformation for differential systems. Pacific J. Math., 8:575-584, 1958. MR 20:5913

[21] W. T. Reid. Generalized polar coordinate transformations for differential systems. Rocky Mountain J. Math., 1(2):383-406, 1971. MR 43:6488

Department of Mathematics and Statistics, University of Missouri-Rolla, 313 Rolla Building, Rolla, Missouri 65409-0020

E-mail address: bohner@umr.edu

Mathematical Institute, Czech Academy of Sciences, ŽižKova 22, CZ-61662 Brno, Czech Republic

E-mail address: dosly@math.muni.cz 